\title{
Dental images - Their use and abuse
}

SADJ November 2020, Vol. 75 No. 10 p 584 - p590

LM Sykes ${ }^{1}$, A Uys $^{2}$, C Bradfield ${ }^{3}$, N van Reede van Oudtshoorn ${ }^{4}$

\section{INTRODUCTION}

Patients' exposure to medical and dental radiographic examination has increased over the years, ${ }^{1}$ with dental $\mathrm{X}$-ray procedures now accounting for almost one-third of all radiographic examinations. ${ }^{2}$ Although they only contribute $2-4 \%$ towards the collective effective dose of exposure, all efforts should be made to minimize the amount taken and to keep exposure as low as diagnostically achievable. ${ }^{2}$

When considering radiographic examinations, the potential diagnostic or therapeutic benefits to the individual or society need to be weighed up against the possible risks that the exposure may cause, taking into account the "efficacy, and benefits and risks of alternative techniques that have the same objectives but involve no or less radiation"., 2,3

To this end the acronym ALARA was coined to stress that all diagnostic radiographs should aim to keep doses as low as reasonably achievable without compromising the diagnosis. ${ }^{1}$ With the advent of digital imaging there has been a trade-off between image quality and reduced radiation dosage. As such the term has been altered to ALADA, as low as diagnostically acceptable, to reflect this compromise. ${ }^{2}$

\section{Literature review}

The best approach to reduce radiation exposure is to follow a strict protocol of justification (considering benefits versus risks), optimization (selection of the best type of radiographic examination) and limitation (implementing radiation protection and minimising exposure). ${ }^{2}$

Author affiliations:

1. Leanne M Sykes: BSc, BDS, MDent, IRENSA, Dip Forensic Path, Dip ESMEA, Head of Department of Prosthodontics, University of Pretoria, South Africa.

ORCID Number: 0000-0002-2002-6238

2. Andre Uys: BBSc, BChD, Dip.Odont. (Materials), Dip.Odont. (Endo), MSc (Odont.), PhD, Department of Oral Pathology and Oral Biology, University of Pretoria, South Africa.

3. Charles Bradfield: $B$ Tech, BChD, Dip Aesthetics, Registrar Department of Prosthodontics, University of Pretoria, South Africa.

4. Nicolaas $\mathbf{W}$ van Reede van Oudtshoorn: $B C h D, P D D$, Private Practitioner, South Africa.

ORCID Number: 0000-0001-5669-795X

Corresponding author: Leanne M Sykes

Department of Prosthodontics, University of Pretoria, South Africa.

Email: leanne.sykes@up.ac.za

Author contributions:

1. Leanne M Sykes: Primary author $-30 \%$

2. Andre Uys: Second author $-30 \%$

3. Charles Bradfield: Registrar - $30 \%$

4. Nicolaas $W$ van Reede van Oudtshoorn: Third author - $10 \%$
This can be achieved in three ways. Firstly, by physically minimising dosage through equipment factors. ${ }^{4}$ However, clinicians who try reduce dosage by altering machine settings must be aware that radiographs obtained with very low dose exposure settings that have no diagnostic value due to poor image quality cannot be justified or ethically condoned. ${ }^{2}$

Secondly they should apply appropriate selection criteria when deciding if radiographic examination is needed. ${ }^{4}$ The routine practice of taking a panoramic radiograph (PR) or full mouth periapical images (PA) for all new patients is not justified, and any radiographs taken should be based on the patient history, clinical examination including a study of previously taken radiographs (if available, recent and of good quality), ${ }^{2,3}$ consideration of all alternative non-radiographic options, ${ }^{2}$ and the determined need. ${ }^{4}$ Thereafter, radiographs should only be taken if they will make a "substantial contribution to distinguishing between treatment options" and/or will provide additional information which could change the diagnosis and management. $^{2}$

Thirdly they should have established quality assurance programmes to ensure that all radiographs taken are of high quality and diagnostic value in order to avoid the need for repeated exposure. ${ }^{4}$ By basing radiographic selection on this strategy of clinical evaluation in asymptomatic patients and selected radiographic imaging in symptomatic patients has resulted in a $43 \%$ reduction in the number of radiographs taken with no corresponding clinical increase in undiagnosed disease. ${ }^{3}$

Special attention should be paid to minimize radiation exposure in vulnerable patients such as children and pregnant patients. Factors that should influence the decision to perform a radiograph should include: patient age, economic indicators, the patient's vulnerability to known risk factors and the medico-legal consequences if disease is undetected and untreated.

Informed consent is needed from all patients prior to exposing them to any radiation procedure. They need to be alerted as to the potential hazards, and where possible X-ray free examination techniques should rather be used during the decision making process. If radiographs are taken, then the dose and diagnosis must be recorded in the record files. This is both a legal requirements and will help avoid extra exposure from repeat examinations. Any clinician who takes a radiograph must be competent and trained in its evaluation and interpretation. This necessitates they have a working 
knowledge of anatomy and pathology, and the ability to detect non-significant artefacts. Where CBCT scans are used they must also be able to identify and interpret 3D structures. In addition, they need to ensure that they analyse the entire field of capture and not just the areas of interest. ${ }^{\star \star 2}$

Kühnisch et al. (2019) and Beneyto et al. (2007) proposed guidelines and clinical indicators for taking radiographs which have been summarised below. ${ }^{2,4}$

\section{Bitewing (BW) radiographs}

It has been suggested that as the caries prevalence has declined in many industrialised countries, so too has the need for BW radiographs. They are still often indicated for children and adolescent patients in order to detect proximal caries in enamel and dentine, occlusal carious lesions, to classify and monitor caries extension, to detect secondary caries, to evaluate the quality of dental restorations, and to assess interproximal bone levels. ${ }^{2}$ Patients with a high caries risk profile may need more frequent monitoring by means of follow up BW radiographs, at individually determined time intervals.

They are not recommended for detecting bone loss associated with periodontitis, ${ }^{2}$ but may be used for patients with uniform pockets less than $6 \mathrm{~mm}$, or irregular shallow pocketing. ${ }^{4}$

\section{Periapical (PA) radiographs}

These are suitable for assessing dental anatomy (root canal morphology, root development, apical areas), and dental pathology (periapical lesions, furcal involvement, dental trauma, and various forms of tooth resorption). They may be used in several clinical situations such as:

- In patients with symptomless, vital teeth with deep carious lesions and / or teeth with symptoms of reversible pulpitis where endodontics or extractions may be needed. ${ }^{2}$

- In symptomatic and / or non-vital teeth with deep carious lesions or other pathoses where the BW is not able to capture the root sufficiently, and to establish the feasibility of endodontic treatment. ${ }^{2}$

- Following dental trauma to detect root fractures and tooth developmental stages (in children). ${ }^{2}$

- In teeth with anatomical malformations or developmental disorders. ${ }^{2}$

- In cases of suspected supernumerary teeth, impactions or retained deciduous teeth. ${ }^{2}$

They are essential for all aspects of endodontics including preoperative views to determine pulp and root canal anatomy and length, working length estimation, during mechanical treatment, and post-operatively to assess the success of the obturation. ${ }^{4}$ They are also mandatory for any surgical root canal treatment.

They are only indicated prior to extracting teeth if there is a history of difficult extractions, where there is a suspicion of complex root anatomy, prior to orthodontic extractions, for lone standing maxillary molars, for teeth with associated swelling, in impacted or partially erupt- ed teeth, for roots that may be lying close to important anatomical structures, and in patients with medical histories that will place them at risk if complications occur. ${ }^{4}$

They may be used in patients with more advanced periodontitis or with suspected periodontal/endodontic lesions. $^{4}$

\section{Panoramic radiographs (PR)}

Routine use of PR as a screening tool is unjustified, as studies have found that over $65 \%$ of these images had no relevance to the subsequent treatment. ${ }^{4}$ They are also not needed in edentulous patients who present with no clinical signs and symptoms unless implant therapy is planned. ${ }^{4}$ In which case a CBCT is the more appropriate modality to use. ${ }^{4}$

They are not suited for initial caries detection or cases of mild acute dental infections. ${ }^{3}$ In more severe cases they can help to view the extent of decay, presence of associated infection, and to decide if the teeth are saveable. They may also be easier to take in situations where there is associated extra/intra oral sepsis, pain and swelling. ${ }^{2}$

They are indicated for dental conditions affecting larger areas of the jaws or situations where a patient cannot tolerate the intra-oral device needed for a BW or PA radiograph. However in the latter situation there will be the trade off in image quality and possible ghost images associated with PR radiographs. ${ }^{2}$

They are essential in cases of trauma to assess jaw and joint fractures. However, PA images are still better for detecting dental fractures, ${ }^{2,4}$ and other views may also be better suited to assessing high condylar fractures. $^{4}$

They may be used to assess the full mouth and jaws in patients who have generalized dental anomalies or congenital defects. ${ }^{2}$

They may be indicated in situations where PA radiographs suggest possible bone pathology, in order to view a wider field and to help diagnose the condition. ${ }^{2}$

They may be used to evaluate impacted third molars prior to planned extraction as they can provide information about tooth position, relationship to the maxillary sinuses (maxilla) and inferior alveolar nerve canal and lower border (mandible). Erupted third molars can usually be assessed with PA radiographs. (Note previous radiographs may reveal that there are no opposing third molars present and negate the need for any radiographs in patients with suspected impactions). ${ }^{4}$

They may be used in certain orthodontics cases to show the state of the dentition, and presence or absence of underlying teeth, but are not always necessary as part of routine treatment. ${ }^{4}$

They may be used in patients with advanced periodontitis and heavily restored dentitions as an alternative to taking many PA images. They are also good for monitor- 
ing the rate of disease progression in these patients. However they may not show as fine detail as the latter and supplementary PA may still be needed. ${ }^{4}$

Although they are often used as an initial screening tool in patients with TMJ symptoms, studies have shown that they provided little information to influence the diagnosis or subsequent treatment. ${ }^{4}$ This may be because a high number of these patients were suffering from myofacial pain/dysfunction or internal disc derangement, and are better diagnosed with magnetic resonance imaging $(\mathrm{MRI}){ }^{4}$

PR can reveal calcifications of the carotid artery by examining the area 1.5 to $2.5 \mathrm{~cm}$ posterior and inferior to the angle of the mandible. However they should not be taken for this purpose alone, but rather note that all routine PR should be evaluated for this condition. ${ }^{3}$ If suspected or found the patient should be referred to a physician for further management. ${ }^{3}$

\section{Cone-beam computed tomography (CBCT)}

CBCT is used to provide detailed cross sectional images of the teeth and surrounding tissues. The field of view (FOV) varies according to the equipment and machine settings and needs to be chosen according to the area of interest in order to keep the exposure dose as low as possible. ${ }^{2}$

CBCT is seldom indicated in children, not used for caries detection, or to detect acute dental infections (unless the aetiology cannot be established with any of the other methods). They are seldom used to identify dental or dento alveolar trauma, except in cases such as where the palatal root of a maxillary molar needs to be more closely examined, but one must be aware that the image quality may be distorted by restorative or root filling materials. ${ }^{2}$ Some clinicians have advocated their use for detecting the extent of lesions associated with invasive cervical resorption. ${ }^{2}$

They may be helpful to establish the location of teeth with eruption disturbances, especially maxillary canines, as well as any other unerupted impacted or ectopic teeth. ${ }^{2}$

They are suited and in fact recommended in all patients where osseiontegrated implant therapy is planned. They allow for accurate assessment of bone quality and quantity, help in the choice of implant type, length and diameter, aid in planning implant location, as well as for fabricating surgical guides and manufacturing immediate/provisional restorative prostheses. ${ }^{2}$

${ }^{*}$ NOTE: If a CBCT scan is taken, the onus is on the clinician to ensure they are interpreted and reported on by competent trained professionals. Additionally they have a duty to evaluate the entire FOV and not only comment on the areas of interest. Failure to do so may hold them liable for clinical negligence. ${ }^{2}$

Having established guidelines and indications for the use of dental radiographs, two clinically related ethico-legal issues arise.
1. Patients where radiographs were taken and not needed for the diagnosis and treatment.

2. Patients where treatment was carried out without the requisite or appropriate radiographs having been taken, or where images were not adequately assessed and interpreted, where an excessive number of images were taken, where an unsuitable view was selected for the specific condition, where images revealed crucial information that was not detected, or where any radiographic errors/omissions resulted in compromised and/or unsuited management.

The following three cases illustrate cases of correct radiation use as well as radiation abuse.

\section{CASE 1 - Justified use of diagnostic aids (Figures 1 to 4 )}

The patient was referred for a panoramic radiograph due to pain affecting the mandible. The dentist detected a radiolucent lesion associated with the mesial root and a loss of lamina dura of the distal root of tooth 47 .

This prompted him to perform vitality tests in which both the 46 and 47 tested vital. In addition, no carious lesions were seen clinically or radiograpically on either tooth. It was thus justified to refer for additional imaging. A small field of view CBCT was exposed to limit the area and radiation dosage.

Three dimensional reconstruction of the image data showed destruction of the buccal cortical plate in relation to the 47 . The axial and coronal CBCT images confirmed the destruction of the buccal cortical plate. The lesion appeared radiolucent and had a resultant change of the normal internal trabecular structures. Once again further investigation was warranted, and a bioposy was taken. The histolopatgological evaluation confirmed the lesion to be an osteosarcoma, which was subsequently managed.

Had the dentist not been vigilant during the intial examination of the panoramic radiograph this serious, condition may have remained undetected, with potentially fatal consequences.

\section{CASE 2 - Unjustified radiographic exposure} (Figure 5)

A 4 yr. old patient referred for a panoramic radiograph. The radiograph was not justified as all interproximal surfaces could have been examined visually, and with minimal discomfort to the child, by careful probing.

CASE 3 - Unjustified exposure where a panoramic radiograph was taken but not examined or interpreted correctly prior to carrying out a full mouth rehabilitation (Figure 6-10).

The patient presented for a routine dental examination complaining of generalised toothache, and missing anterior teeth (12 and 13). A panoramic radiograph was taken as well as numerous "before" (and after) photographs showing a heavily restored dentition (Figures 6, 7 and 8). 


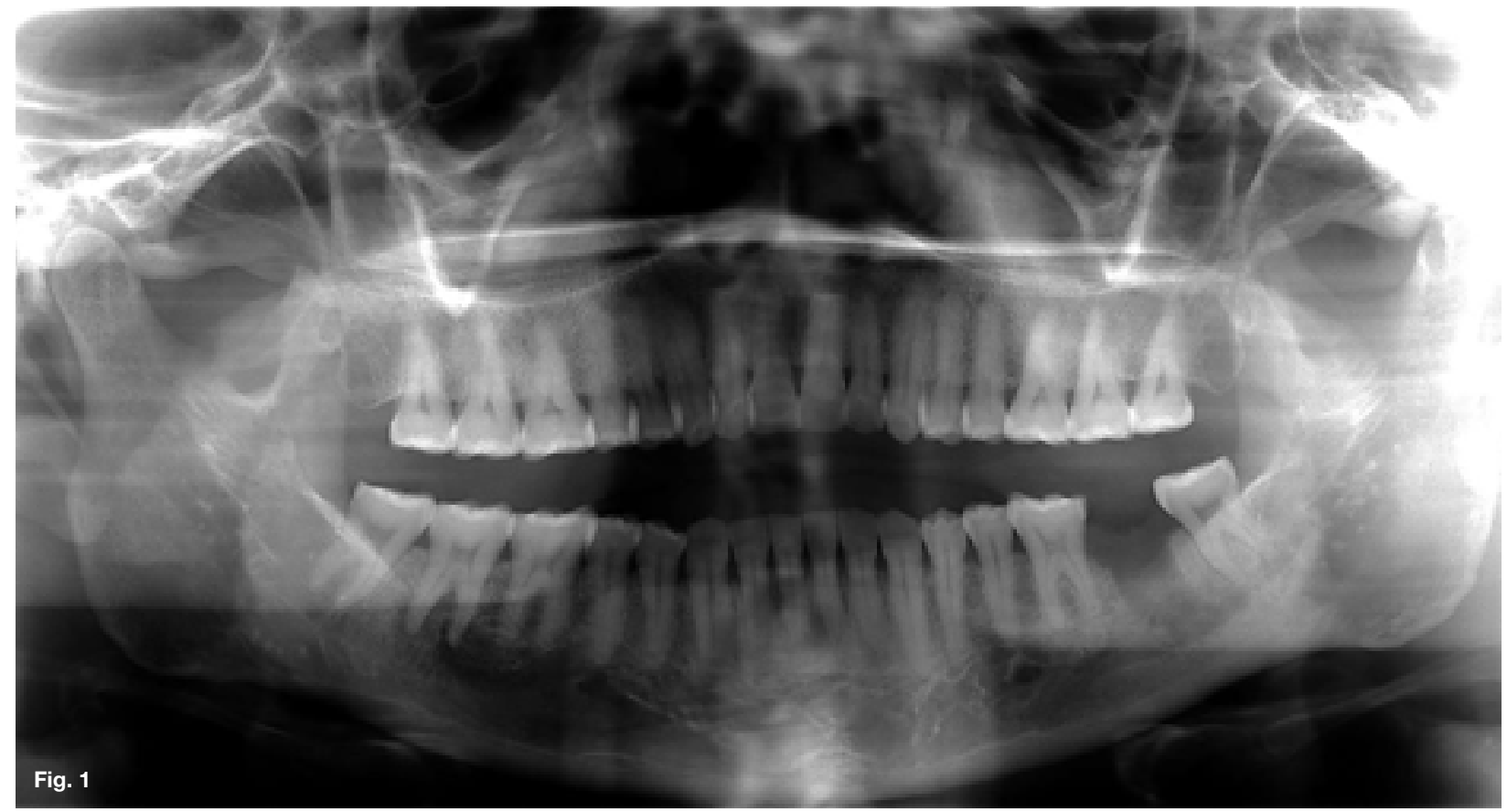

Figure 1. Panoramic radiograph.

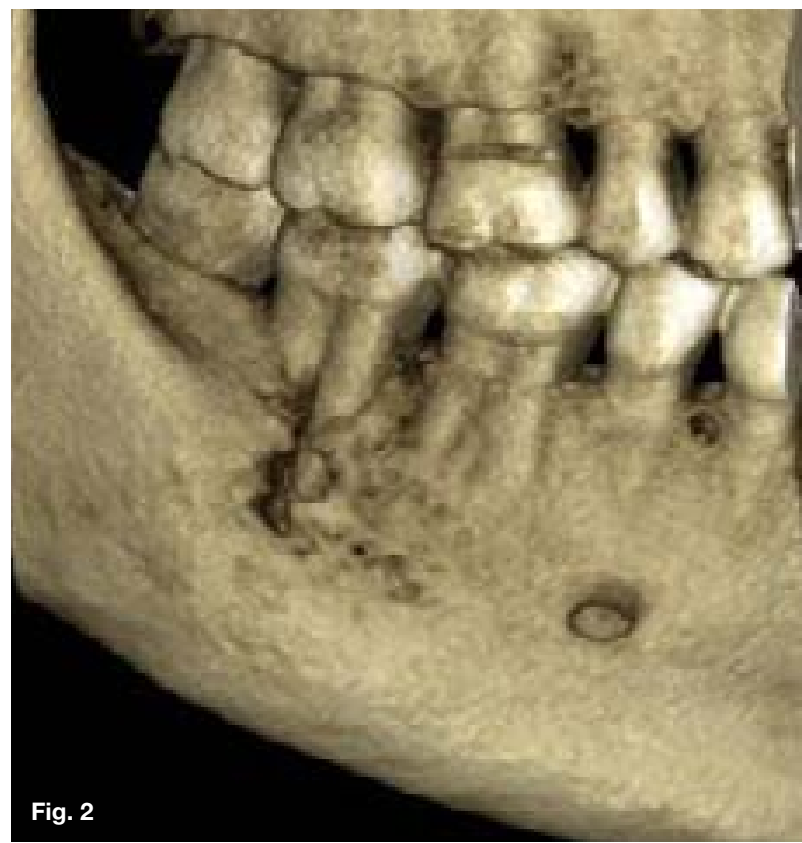

Figure 2. Three dimensional reconstruction of the image data showing destruction of the buccal cortical plate in relation with tooth 47 .

The dentist, an "expert and trainer in CAD/CAM technology" informed her that many of her fillings were failing and should be replaced. He also indicated that numerous other teeth needed to be restored. He suggested that all of her restorations be replaced with "white crowns", as well as full coverage crowns on many of her remaining teeth to create a "perfect smile".

The teeth were all prepared, scanned and restored the same day with CAD/CAM fabricated full coverage crowns, and a 4-unit bridge from the 14 to the 11. In total she was provided with 10 crowns and a 4-unit bridge in the maxilla and 7 crowns in the mandible (In total, 17 crowns, one of which was implant supported, and 2 pontics). At the same visit the scaling and polishing was done to remove calculus and staining of

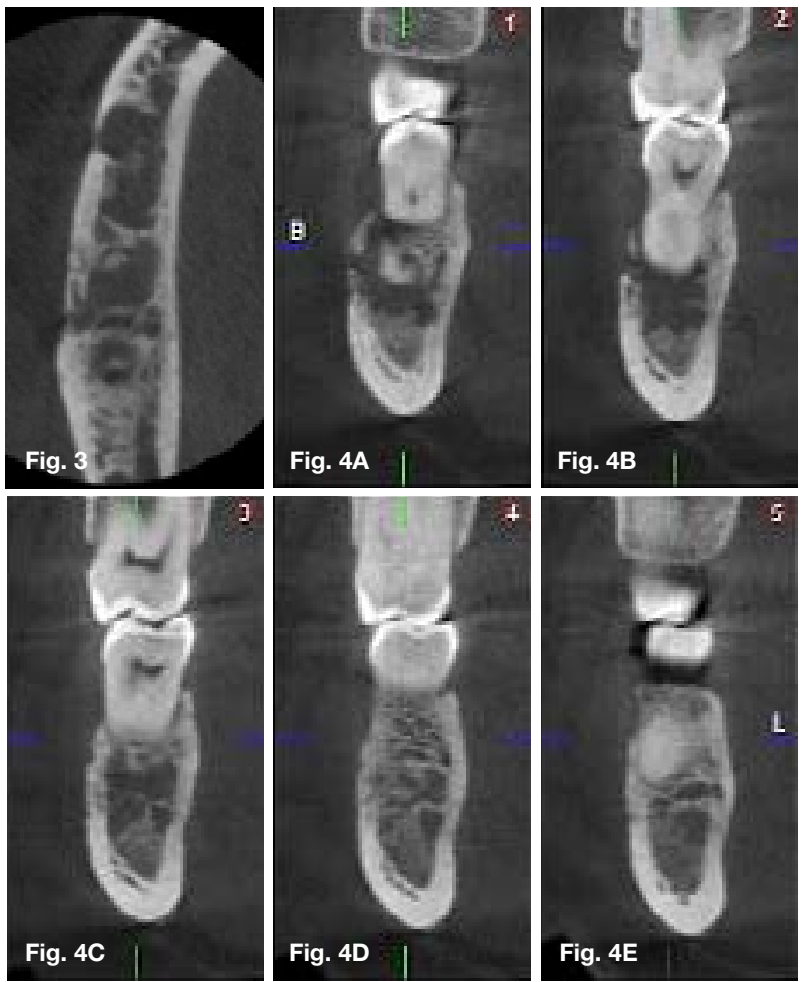

Figure 3 and 4. Axial and coronal CBCT images showing destruction of the buccal cortical plate

the mandibular anterior teeth. The total cost of treatment exceeded R400 000.00 (2018 rates).

She experienced generalised ongoing sensitivity after completion of the work, which her dentist reassured her would dissipate. However, after a few months of constant pain, and sensitivity she sought a second opinion.

Dentist 2 requested her pre-treatment records from her original dentist and was provided with a number of intra oral pre and post-operative photographs, and a $\mathrm{PR}$ radiograph. There were no PA views available to 
$588>$ ETHICS

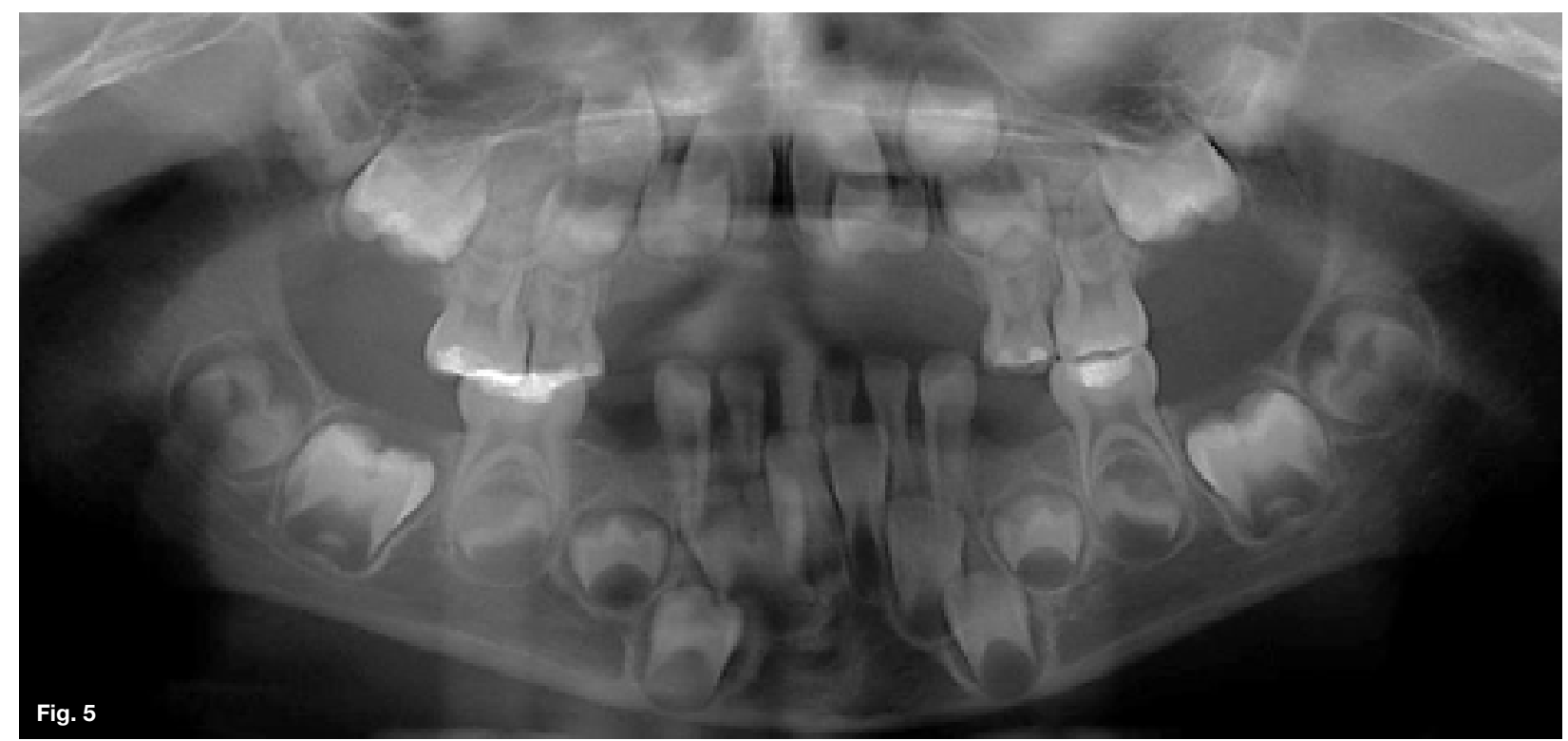

Figure 5. A panoramic radiograph of a 4-year-old child.

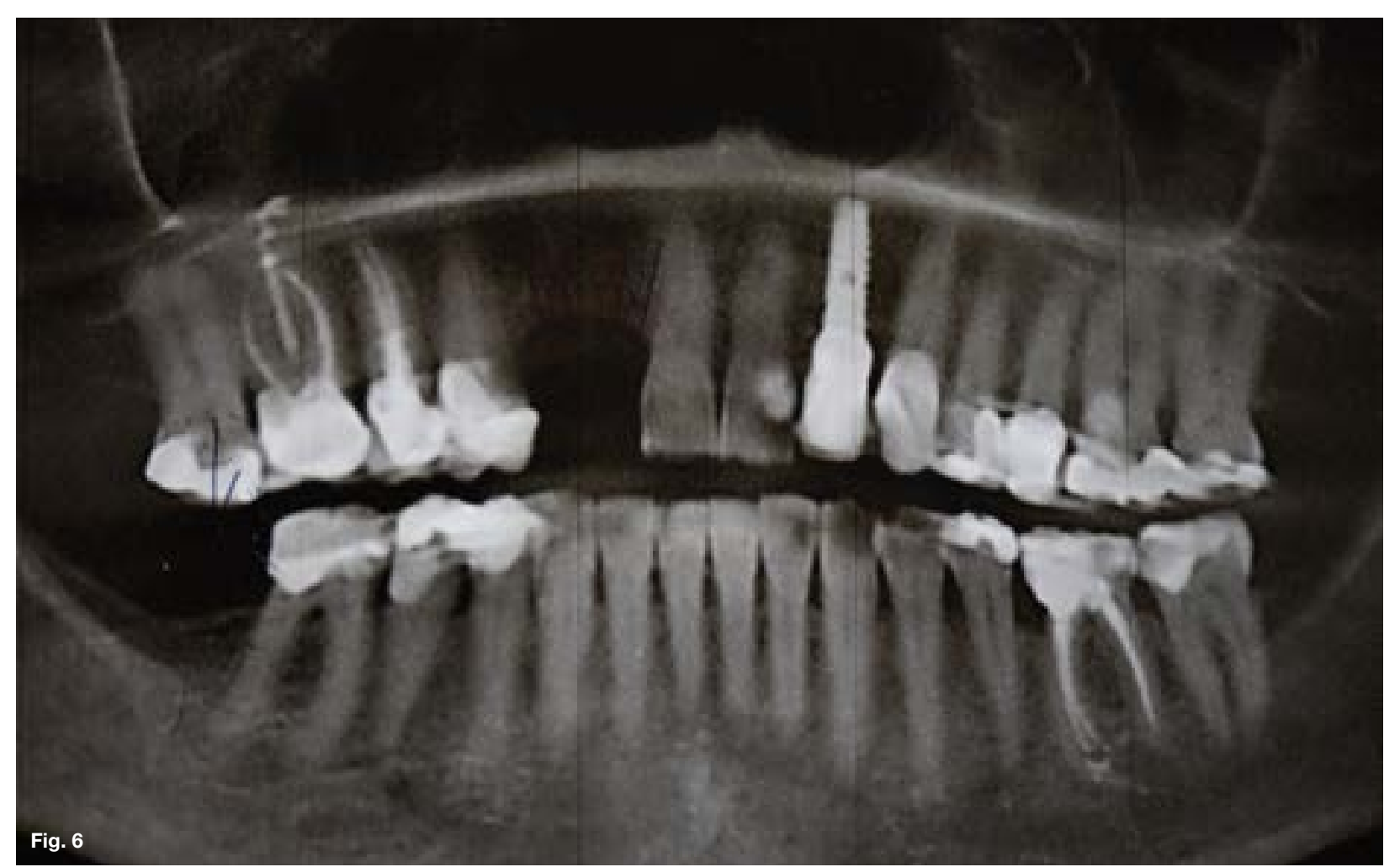

Figure 6. Pre-treatment panoramic radiograph.

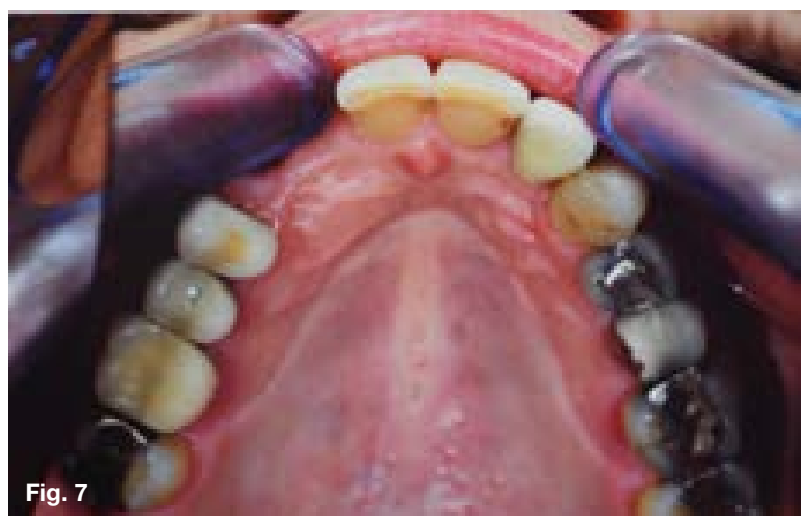

Figure 7. Pre-treatment occlusal view of maxillary arch.

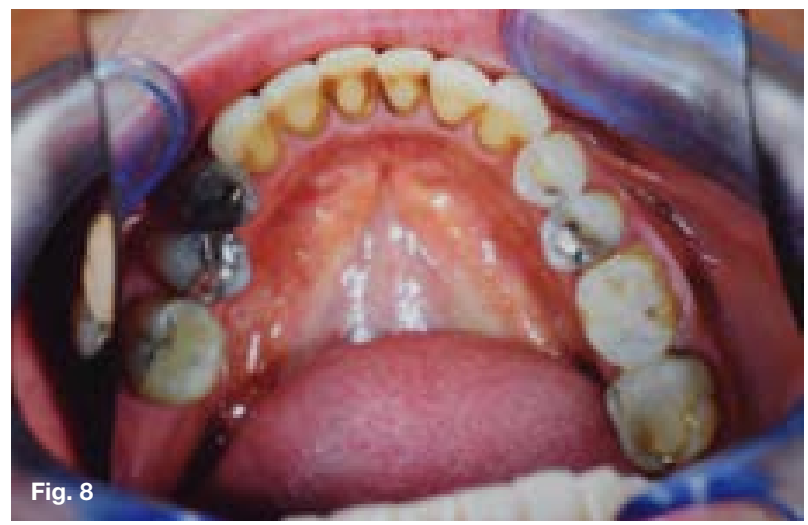

Figure 8. Pre-treatment occlusal view of mandibular arch. 


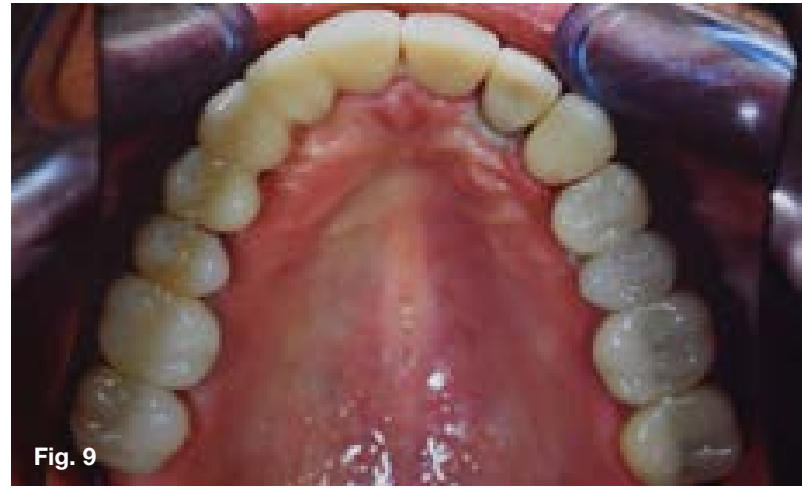

Figure 9. Post-treatment occlusal view of maxillary arch.

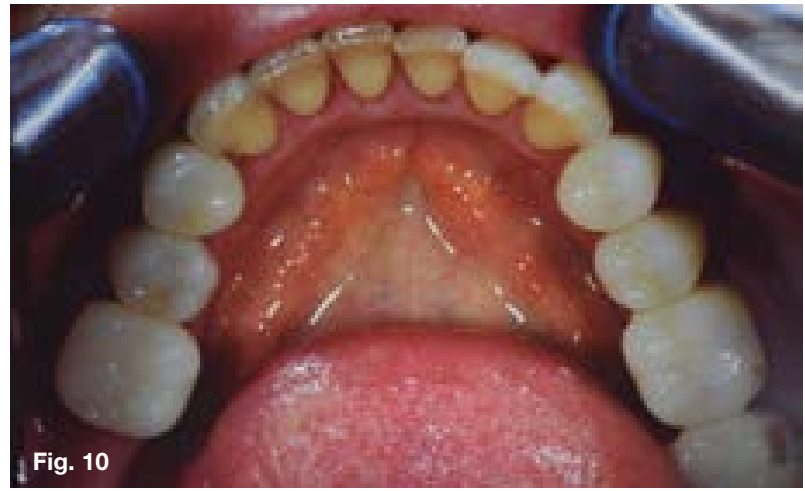

Figure 10. Post-treatment occlusal view of mandibular arch.

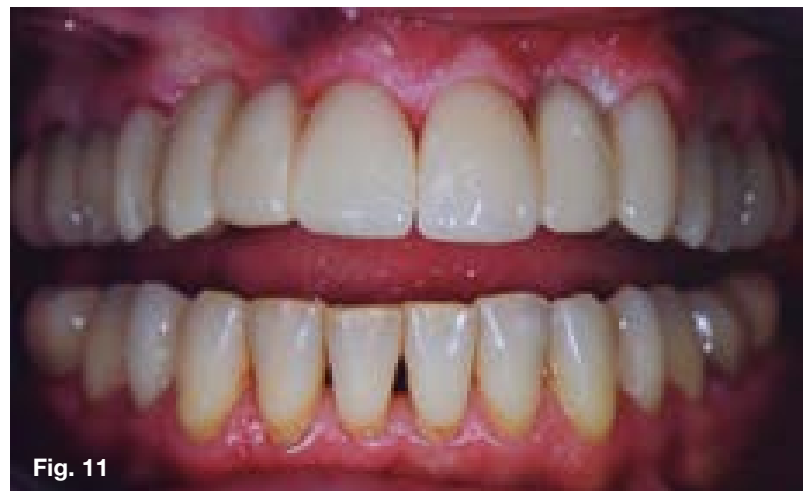

Figure 11. Post treatment rehabilitation. indicate that there had been a tooth-by-tooth analysis. $\mathrm{He}$ examined this data closely. A detailed analysis of the panoramic radiograph revealed the following: generalized moderate to severe bone loss, particularly in the posterior segments with furcation involvement of the 36 and 46 . There was also extruded root filling material and a periapical radiolucency on the 16 .

Teeth $17,16,26,37,36,45$ and 46 were all considered to be in need of a periodontal assessment, diagnosis and treatment before any fixed restorative work was done in order arrest the bone loss, and restore her mouth to health. Large and/or faulty restorations with deep subgingival margins were noted on the $16,15,25,26,27,36,45$ and 46.

The need for replacing these would have to be confirmed with a clinical evaluation and PA radiographs. She was also in need of a 4-unit bridge spanning from 14 to 11. Many of these teeth may not have needed treatment or could potentially have been restored with small restorations. Thus the treatment plan should have consisted of initial periodontal therapy, extraction of unsaveable teeth (36 and 46), crowns on selected teeth, a 4-unit bridge and minor restorative work. Dentist 2 also took a follow up panoramic radiograph which confirmed that the patient had never received the requisite periodontal therapy prior to this extensive rehabilitation (Figure 12).

This case is a clear example of radiographic abuse, gross over servicing and arguable malpractice. Not only were restorations placed on teeth that were unquestionably in need of periodontal treatment, but also on teeth with minor carious lesions.

She had in effect been provided with an extensive rehabilitation, yet there was no record of any occlusal analysis having been performed prior to cutting the teeth, nor any consideration of placing her in provisional restorations for a time in order to monitor and evaluate the new vertical dimensions, occlusal scheme, aesthetics and speech and masticatory functions.

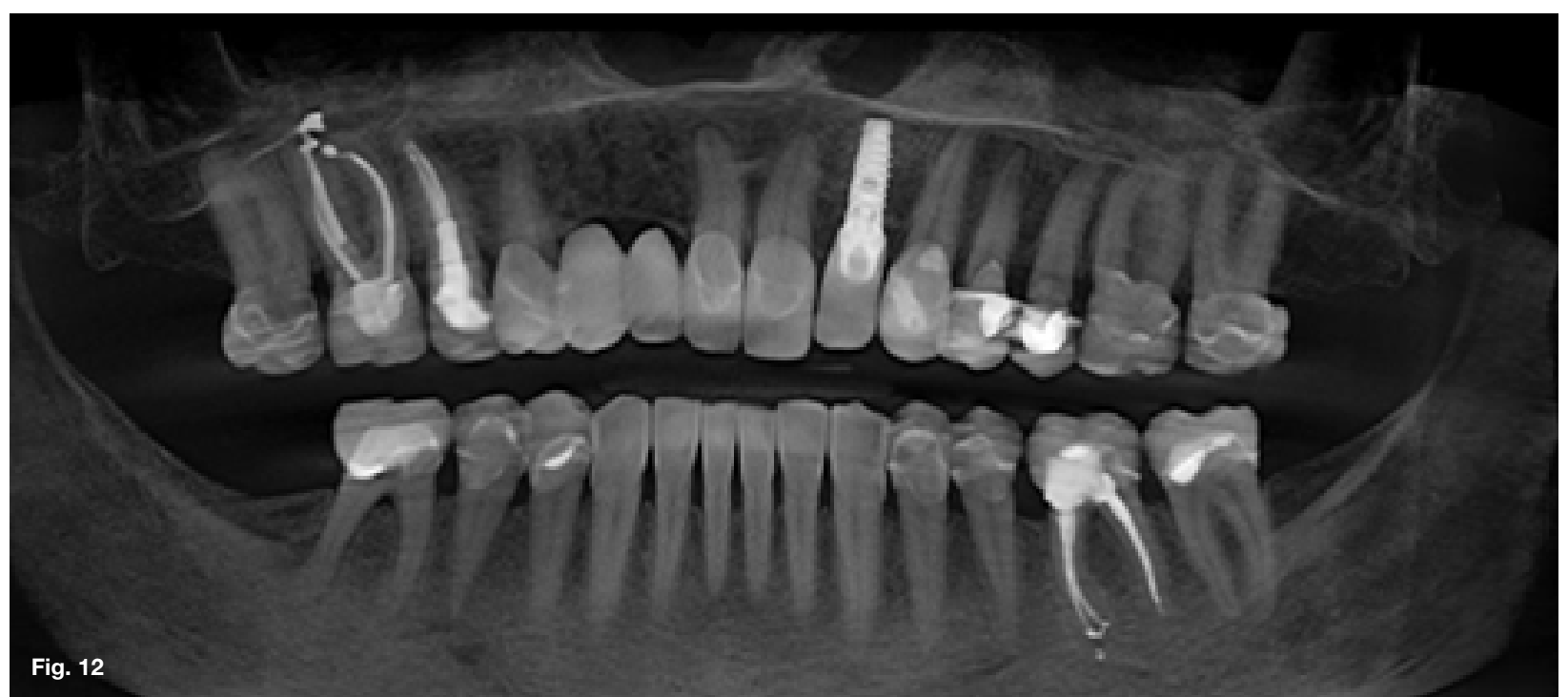

Figure 12. Post treatment panoramic radiograph revealing persistent periodontally compromised dentition. 


\section{DISCUSSION}

Diagnostic aids may take the form of photographs, study models, radiographs, clinical observations and measurements, microbial tests, or pathologic/histological investigations. They are an extremely useful, and often crucial part of the initial patient examination and treatment planning appointment. However, their value depends on the correct choice, evaluation, interpretation, diagnosis, and subsequent implementation of management and treatment procedures. Radiographs are one of the most widely used diagnostic aids in dentistry due to their ability to reveal structures and conditions that are impossible to visualise or detect clinically. However, they carry associated financial costs and patient risk factors. Taking incorrect views, exposing patients to unnecessary or excessive amounts of $X$-rays, repeating procedures when not needed, or failure to examine and interpret the radiographs correctly is not only unethical but borders on negligence and malpractice.

\section{CONCLUSION}

The cases presented in this paper illustrate three situations where panoramic radiographs were taken. In the first, the dentist carried out a detailed and meticulous radiographic evaluation, and was astute enough to request further diagnostic procedures, using the correct modality (sectional CBCT). This revealed a potential life threatening condition and resulted in early and appropriate patient management. In the second case, it was unjustified to expose a young patient to this radiation when a clinical examination would have sufficed. In the third situation, not only did the dentist expose and charge the patient for the radiographs, but the subsequent treatment was carried out with no consideration of the dental conditions revealed in the radiograph. This resulted in gross over servicing with unsuitable treatment that could be considered medical malpractice. If a clinician does not have the expertise to analyse a particular image then they should not make use of that modality. However, this option could be detrimental to the patient's welfare. The more responsible and professional approach would be to develop themselves by acquiring training in this field, or alternatively consulting with colleagues who have the requisite skills and expertise.

\section{"You see only what you look for, you recognise only what you know" - Dr Yvette Solomons}

\section{References}

1. Holmboe E, Bernabeo E. The "special obligation" of the modern Hippocratic Oath for the $21^{\text {st }}$ century medicine. Med Edu. 2014; 48(1): 87-942.

2. Davey LM. The oath of Hippocrates: an historical review. Neurosurg. 2001; 49: 554-663.

3. Sulmasy D. What is an oath and why should physician swear one? Theor Med Bioeth. 1999; 20: 329-46.

4. Steihm ER. Adverse effects of human immunoglobulin therapy. Transfus Med Rev. 2013; 27: 171-8.

5. Legnani $P$, et al. Atmospheric contamination during dental procedures. Quint Int. 1994; 25: 435-9.

6. Checchi $V$ et al. COVID-19 dentistry related aspects: a literature review. International Dental Journal. 2020. https:// doi.org/10.1111/idj.12601.
7. Ather A, et al. Coronavirus disease 19 (COVID-19): implications for dental care. Journal of Endodontics. 2020; 46(9): 1341-2.

8. Ge Z, et al. Possible aerosol transmission of COVID-19 and special precautions in dentistry. Journal of Zhejiang University - SCIENCE B. 2020; 1- 8. doi.org/10.1631/jzus. B2010010.

9. Xiao A, et al. Dynamic profile of RT-PCR findings from 301 COVID-19 patients in Wuhan, China: a descriptive study. Journal of Clinical Virology. 2020; doi.org/10.1016/j.jcv.2020. 104346.

10. Watkin, et al. Top E.R Doctor Who Treated Virus Patients Dies by Suicide. The New York Times. Available at: https:// www.nytimes.com/2020/04/27/nyregion/new-york-citydoctor-suicide-coronavirus.html/ (Accessed: 19 June 2020).

11. Sokol DK. Virulent epidemics and the scope of healthcare worker's duty of care. Emerg Infect Dis. 2006; 12(8): 1238-41. doi:10.3201/eid1208.060360.

12. Ruderman $C$, et al. On epidemics and the duty to care: whose duty? who care? BMC Ethics 2006; 7(1): 1-6.

13. Deanery $S$, et al. Healthcare worker's perception of duty to work during influenza pandemic. J Med Ethics. 2010; 36(1): 12-18.

14. Jeffrey DI. Relational ethical approach to the COVID-19 pandemic. J Med Ethics. 2020; 0: 1-4.

15. Hruschka, J. The greatest happiness principle and other early German anticipations of utilitarian theory. Utilitas. 1991; 3(2): 165-77.

16. Singer PA, et al. Ethics and SARS: Lessons from Toronto. BMJ. 2003; 327: 1342-4.

17. Dwyer J, Tsai DF. Developing the duty to treat: HIV, SARS and the next epidemic. J Med Ethics. 2008; 34(1): 7-10.

18. Beauchamp T, Childress J. Principles of biomedical ethics, $2^{\text {nd }}$ ed. New York: Oxford University Press, 1983.

19. Aristotle. Nichomachean ethics. In: McKeon R, ed. The basic works of Aristotle. New York: Random House, 1941.

20. Tomlinson T. Caring for risky patient's duty or virtue? J Med Ethics. 2008; 34(6): 458-62. 